\title{
In Ricordo di Umberto Buoncristiani
}

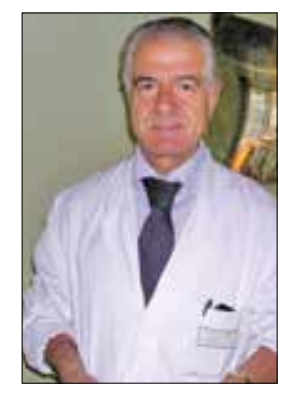

Umberto Buoncristiani

Umberto, Addio

Per me Umberto era un fratello e il lato affettivo superava di gran lunga la collaborazione stretta che si è svolta per più di trent'anni tra le nefrologie di Perugia e di Siena.

Corsi, congressi, ricerche e pubblicazioni hanno cementato certamente un'amicizia particolare e quanto mai fruttuosa.

Ma il lato affettivo, lo ripeto, predominava su tutto il resto e coinvolgeva le nostre vite, i nostri sentimenti, le nostre gioie e i nostri dolori.

L’amorevole cura per la sua famiglia è rimasta sempre al di sopra del lavoro e della ricerca che, da soli, occupavano già molte ore della sua vita.

Ricorderò sempre quello che mi raccontò una sera in attesa di imbarcarsi su un aereo per gli Stati Uniti; mi disse che la professione e le sperimentazioni lo impegnavano così tanto che la sera tornava a casa sempre stanco e molto tardi, ma la dolce buonanotte dei suoi figli lo ripagava dei sacrifici; a casa, poi, lo attendeva anche la sua sposa che, mentre lui cenava, si metteva al pianoforte e suonava dolci melodie che vincevano del tutto la stanchezza e lo stress accumulati nella giornata.

Umberto Buoncristiani era un vero Signore e come tale si comportava sia nel lavoro che nella vita sociale. Non l'ho mai sentito alzare la voce, imprecare contro qualcuno o pretendere qualcosa da chicchessia. Aveva sempre parole di incoraggiamento e di speranza verso i suoi malati ed era una forza trainante verso i suoi collaboratori e, per questo, tutti lo rispettavano, sia i colleghi che i pazienti, sorretti dalla fiducia e dalla forza di volontà che metteva in ogni sua manifestazione.

L'improvvisa e tragica fine della sua diletta primogenita lo annichilì e lo sconvolse nel profondo, ma non lo distrusse. Riuscì a reagire riversando sulla moglie e sugli altri due figli tutto l'amore che aveva riservato alla prima.

Umberto era indubbiamente bravo come medico ed era intelligente come ricercatore; la storia della Nefrologia italiana è costellata di sue realizzazioni. Era un insegnante perfetto, perché riusciva a trasmettere agli allievi la forza delle sue realizzazioni e la comprensione del suo pensiero scientifico.

Umberto e io abbiamo avuto modo di viaggiare molto insieme perché invitati entrambi a parlare di dialisi peritoneale a tanti congressi in tutto il mondo; per me è stato sempre un grande piacere spostarmi con lui anche in luoghi lontani. II suo notevole bagaglio culturale e la sua affettività rendevano piacevoli le attese, anche le più esasperate.

La sua tragica scomparsa ha creato un vuoto incolmabile nella Nefrologia italiana e una ferita molto dolorosa nel mio cuore.

\section{Nicola Di Paolo}

L'11 Maggio 2016 è venuto a mancare Umberto Buoncristiani, punto di riferimento delle nefrologia e primo responsabile dell'Unità di Nefrologia Dialisi e Trapianto di Perugia.

Un male incurabile veloce e subdolo ha portato via Umberto Buoncristiani alla sua famiglia e all'effetto dei suoi cari. Buoncristiani è stato un nefrologo di fama mondiale che ha creato la nefrologia in Umbria, partendo prima come assistente del Prof. Gigli e, poi, diventando primario del Servizio di Dialisi di Perugia.

Da allora, ha fatto crescere il centro, facendolo dapprima assurgere a Struttura complessa di Nefrologia e Dialisi con letti propri e, poi, permettendo, incentivando e creando il programma di trapianto renale in Umbria, che ha consentito a centinaia di pazienti umbri, e non solo, di abbandonare la macchina per una vita migliore e di maggior profilo.

Ha introdotto, per primo in Umbria e tra i primi in Italia, la tecnica dialitica, che ha consentito di vivere a migliaia di persone e ha migliorato la terapia domiciliare con innovazioni tali da essere considerato uno dei più importanti nefrologi 
internazionali esperti di dialisi, permettendo l'applicazione di sistemi e modalità che ancora oggi tutto il mondo usa, facilitando la qualità della vita a moltissimi dializzati.

Ha contribuito in prima persona all'affermazione della scuola di specializzazione di Perugia, così tanto apprezzata che molti dei giovani nefrologi formati a Perugia hanno trovato collocazione in prestigiosi centri nefrologici nazionali fuori dai confini umbri e internazionali.

I nefrologi lo ricordano per la creazione del "set $a Y$ " in dialisi peritoneale, rilanciando tale metodica e facendola diventare uno standard, per le prime sperimentazioni della dialisi senza acetato e per la dialisi domiciliare e quotidiana.

Le sue geniali intuizioni sono ancora attuali e sono ancora alla base di nuove sperimentazioni, come per le apparecchiature di dialisi domiciliare quotidiana, per esempio; i suoi fondamentali contributi sono, tutt'oggi, ripetutamente citati nella letteratura internazionale.

Ma sarebbe ingiusto non parlare di altri aspetti del suo operato, che lo hanno reso simpatico e conosciuto nel mondo nefrologico sia medico che infermieristico. Sua, con il collega Nicola Di Paolo, è stata l'idea del Corso di Aggiornamento in Tecniche Nefrologiche e Dialitiche che distribuiva, come atti dei convegni, libri diventati miti e che si svolgeva ad anni alterni tra Perugia e Siena. Sono rimaste leggendarie le partecipazioni degli infermieri da tutta Italia a centinaia, primo esempio di coinvolgimento e partecipazione attiva alla cura nefrologica di tutto lo staff sanitario, così come sono rimasti mitici i momenti conviviali che accompagnavano questi eventi e che lo hanno sempre visto accanto all'adorata moglie Renata.

Al convegno e ai libri si affiancò presto la rivista di tecniche nefrologiche e dialisi ora diretta da Marco Lombardi, che ha saputo conservare e preservare un patrimonio di conoscenza e innovazione importante e innovativo per il periodo in cui si svolgeva.

Purtroppo, invece, il corso non è stato salvato, disperdendo un patrocinio di cultura e di tradizione unica; fortunatamente il testimone è stato raccolto dall'ANTE che annualmente tiene un corso con lo stesso format di quelli di Perugia e Siena, che riscuote tanto successo.

Come tutte le persone eccellenti ha dovuto lottare perché la vita non è stata generosa con lui, colpendolo nei suoi affetti più intimi con la perdita della figlia Cristina. Ha superato con forza e dignità questi momenti e ha continuato nella sua missione per il bene comune.

È stato vicino alla moglie Renata e vicino a Lei ha passato le ultime ore, testimoniandole, ancora una volta, il suo affetto e il profondo legame.

In sintesi, ha creato la nefrologia umbra con riconoscimenti nazionali e internazionali di alto livello, testimoniati non solo dall'alto numero di pubblicazioni su riviste scientifiche al top del grading mondiale (alcuni dei suoi lavori sono entrati nel gruppo alla base delle Linee Guida americane e italiane sulla dialisi), ma anche dai premi internazionali assegnati alla carriera, premi, riconoscimenti e apprezzamenti che, evidentemente, non gli hanno permesso di evitare le amarezze e la delusione e la tristezza dell'ultima parte della carriera quando, massimo esperto di dialisi, si è visto smembrare il reparto da lui diretto e togliere la responsabilità della sezione di dialisi.

La sua perdita colpisce profondamente pazienti, colleghi e nefrologi italiani, ma, soprattutto, chi, come noi, è nato professionalmente con lui e grazie a lui è cresciuto come nefrologo. Abbiamo iniziato giovanissimi e, tra baruffe, incoraggiamenti e delusioni, ci ha permesso di conoscere la nefrologia spronandoci a fare sempre meglio e, sicuramente, dobbiamo ai suoi insegnamenti, alle sue invenzioni e alla sua collaborazione la nostra carriera scientifica e di prestigio nazionale.

Lo ricordiamo con affetto e riconosciamo in lui colui che ha creato e spinto sempre verso il passo successivo ogni iniziativa, non accontentandosi mai dell'ottenuto, ma cercando sempre il nuovo e il migliore. Alle volte ci è riuscito, altre volte no, altre volte ancora solo in parte, ma ha sempre e comunque voluto costruire, riuscendo a fare della sua struttura uno dei punti di riferimento scientifico internazionale tra i più importanti nel panorama nefrologico.

Ritengo che a Umberto Buoncristiani sia dovuto il ringraziamento di tutti, istituzioni comprese, per la stima, la considerazione e l'impegno profusi, che hanno portato alla crescita nefrologica nazionale e l'Umbria e Perugia a essere conosciute e apprezzate nei circoli scientifici nefrologici di tutto il mondo.

Esprimiamo, a nome mio personale e della Società Italiana di Nefrologia, che, tramite il presidente Santoro, ha voluto esprimere la sua vicinanza, le condoglianze alla sua adorata Renata e ai figli, ricordandolo con affetto.

\section{Pino Quintaliani}


Ricordo Umberto Buoncristiani,

Fondatore, insieme a Nicola Di Paolo, di questa rivista, che viene letta da 28 anni da nefrologi, infermieri, tecnici di dialisi e pazienti.

Lo ricordo come un "Signore" riservato, ma che sapeva essere anche molto alla mano con tutti, nonostante il prestigio raggiunto che, chi ha letto di nefrologia, non poteva non riconoscergli.

Lui e il suo alter ego, con il quale ha creato il sodalizio e l'asse nefrologico dell'Italia centrale, sono stati, per fantasia e immaginazione scientifiche (una dote assai rara e, ancora di più, oggi) prototipici del "Nefrologo Leonardesco".

Credo che personaggi come questi (certo, in Italia ne abbiamo avuti a tutte le latitudini) non abbiano potuto che esserci invidiati dal resto del mondo accademico e, per coloro che hanno potuto condividere con loro gioie e fatiche, è stato un grande e sincero onore percorrere anche solo brevi pezzi di strada... magari tenuti per mano da siffatti maestri.

Quando ho saputo dall'amico Pino Quintaliani della scomparsa di Umberto ho capito immediatamente che veniva a mancare un pezzo di storia dell'italica nefrologia e che un vuoto vero mi (ci) accompagnerà.

A Lui il mio ricordo e la mia gratitudine per ciò che mi ha insegnato direttamente o anche e solo indirettamente studiando i suoi testi.

Marco Lombardi

Editor-in-Chief 